\title{
Testicular liposarcoma: a rare form of testicular
} cancer

\begin{abstract}
Testicular liposarcoma is a very uncommon pathology associated with testicular cancer. Paratesticular soft tissue tumors are rare entities with malignant subtypes accounting for $30 \%$ of cases. ${ }^{2}$ Often misdiagnosed, testicular liposarcoma must be considered in the differential diagnosis of a groin mass. ${ }^{1}$ There are only few cases of the giant testicular liposarcoma of more than $10 \mathrm{~cm}$ reported in the literature. We present the case of a $62 \mathrm{yr}$ old male who presented to urology for a mass in the groin which was thought to be an inguinal hernia. The patient had some associated testicular pain therefore an ultrasound was performed identifying a $7.1 \times 1.5 \mathrm{~cm}$ mass. He underwent a left orchiectomy with removal of the associated mass which showed a well differentiated liposarcoma of a sclerosing variant. The surgical margins were negative however; the patient did undergo a metastatic workup with a $\mathrm{CT}$ of abdomen and pelvis that was unremarkable.
\end{abstract}

Keywords: liposarcoma, hypertension, inguinal hernia, sclerosing type
Volume II Issue 3 - 2020

\author{
Chintav Shah,' Simeon Jaggenauth, ${ }^{2}$ Patrick \\ Pathappillil, ${ }^{3}$ Rishav Sinha ${ }^{3}$ \\ 'Department of Internal Medicine, University of Arkansas for \\ Medical Sciences, USA \\ ${ }^{2}$ Landmark Cancer Center, Arkansas, USA \\ 3Medical student, USA
}

\section{Correspondence: Chintav Shah, Department of Internal Medicine, University of Arkansas for Medical Sciences,} Fayetteville,Arkansas, USA, Email chintavshah5@gmail.com

Received: May 19, 2020 | Published: June 05, 2020

\section{Introduction}

Liposarcoma accounts for approximately $20 \%$ of all the sarcomas; but, it is an extremely rare occurrence in the paratesticular region. ${ }^{3}$ Testicular liposarcoma are a very rare variant of genitourinary malignancies occurring more often in the spermatic cord where it accounts for less than $12 \%$ of all liposarcomas. ${ }^{1,45}$ Liposarcomas of the spermatic cord usually begin to grow directly below the external inguinal ring, so as the tumor becomes enlarged, it presents as scrotal mass rather than an inguinal mass. ${ }^{6}$ Due to the scarcity of cases, there is no consensus on the best treatment and this entity is still very challenging for clinicians in terms of management. ${ }^{1}$ In this paper, we present the case of well differentiated sclerosing type liposarcoma of the left testes. We are writing this case as only a few cases of testicular liposarcoma of the sclerosing type has been reported in the literature. ${ }^{1}$

\section{Case report}

This is a $62 \mathrm{yr}$ old male with a history of hypertension and hyperlipidemia that initially presented to the urologist for swelling in a left groin that was thought to be an inguinal hernia. There was also testicular pain associated with it. Ultrasound of the testes was done that demonstrated evidence of a $7.1 \mathrm{~cm} \times 1.5 \mathrm{~cm}$ non-vascular presumed fluid collection identified superior to the left testicle immediately adjacent to the inguinal canal with a $3 \mathrm{~mm}$ left epididymal cyst also noted. The initial differential diagnosis was either a fat containing hernia versus lipoma. Given these findings, he underwent a left orchiectomy and removal of the associated mass which was found to be consistent with a well differentiated liposarcoma, sclerosing variant. Tumor site involved the spermatic cord measuring $15 \times 12 \times 7.2 \mathrm{~cm}$ with the tumor focally extending to the inked surface of the specimen and the spermatic cord margin appearing negative. The testicle was unremarkable. The tumor appeared to involve the spermatic cord but no involvement of the testicular parenchyma was seen and it focally extended to the peripheral inked margin. Patient was referred to the oncology clinic for the evaluation and treatment options.

Based on the above findings, it appeared that he had a well differentiated liposarcoma which has been completely excised.
Margins were essentially negative but it did approximate the inked peripheral margin. He had $\mathrm{CT}$ of the abdomen pelvis performed demonstrating no evidence of the metastatic disease with post surgical changes present in the left lower quadrant superior to the inguinal canal. A small fat filled right inguinal hernia was also seen. Due to these findings, a decision was made to do active surveillance with CT scan of the abdomen/pelvis every 6months.

The patient was followed over the next 18 months with CT abdomen and pelvis which remained negative for any metastatic disease. Patient continues to do well on observation and he is now being followed on a yearly basis with a CT abdomen and pelvis.

\section{Discussion}

Men in their sixth decade exhibit the peak incidence of the testicular sarcomas and usually present as painful or painless mass or swelling, occasionally accompanied by a hydrocoele. ${ }^{2}$ Rhabdomyosarcomas have the propensity to affect the males in the first 2 decades of life and tend to be a more aggressive of the subtype. Differentiating between a benign and malignant lesion by clinical examination alone is not sufficient. ${ }^{2}$ Testicular liposarcomas account for approximately 7 to $10 \%$ of all of the intrascrotal tumors, and have their mesodermal origin arise from adipose tissue. ${ }^{1}$ The most common site of the origin is the spermatic cord which accounts for $90 \%$ of the cases. ${ }^{1}$ There are 5 types of histological subtypes of liposarcoma according to WHO Classification: ${ }^{7}$
a. Well differentiated tumor
b. Atypical lipomatous tumor
c. Dedifferentiated tumor
d. Myxoid tumor
e. Pleomorphic tumor

The most common test used to diagnose is an ultrasound; however, MRI and CT scans may also be used. ${ }^{2,8}$ The imaging studies are important due to the radical nature of the resection in the malignant 
cases. ${ }^{2}$ Due to the rarity of this kind of cancer, knowledge has only been limited to the case reports. 1 The most widely used treatment approach include: Radical Orchiectomy with high ligation of the spermatic cord at the inguinal canal. ${ }^{1,9}$ Incomplete excision is associated with frequent recurrences. ${ }^{10}$ The $3 \mathrm{yr}$ local recurrence free survival was $100 \%$ with negative margins compared to $29 \%$ with for positive margins. ${ }^{11}$ Retroperitoneal lymph node dissection should be limited to patients with only radiologically suspicious lymph nodes. ${ }^{1,2,12}$ Indications for adjuvant radiotherapy includes positive margins or less than $10 \mathrm{~mm}$ and when the tumor is not resectable, because local recurrence following surgery alone is very high. ${ }^{13,14}$ The prognosis depends on the histological cell type. The well differentiated types have a better prognosis but tend to have a high incidence of local recurrence. ${ }^{15}$ Due to the paucity of the cases the role of adjuvant systemic chemotherapy is not clear. When chemotherapy is indicated, treatment with vincristine, cyclophosphamide and doxorubicin is used for high grade and metastatic testicular liposarcoma. ${ }^{16}$

\section{Acknowledgments}

None.

\section{Conflicts of interest}

The authors declare there is no conflict of interests regarding the publication of this paper.

\section{Funding}

None.

\section{References}

1. Mouden K, Soukaina W, Afaf S. Paratesticular Liposarcoma: a Case Report. Pan Afr Med J. 2019;33:282.

2. Robert AK, Riogh A, Stroiescu A, et al. Paratesticular Sarcomas: a Case Series and Literature Review. Ther Adv Urol. 2019;11:1756287218818029.

3. Stefano C, Luciani LG, Cai T, et al. Unusual Case of Locally Advanced and Metastatic Paratesticular Liposarcoma: a Case Report and Review of the Literature. Arch Ital Urol Androl. 2015;87(1):87-89.
4. Noguchi H, Naomoto Y, Haisa M, et al. Retroperitoneal liposarcoma presenting a indirect inguinal hernia. Acta Med Okayama. 2001;55(1):5154.

5. Unlü Y, Gülben EH, Gülzade O, et al. Paratesticular Sarcomas: A Report of Seven Cases. Oncol Lett. 2015;9(1):308-312.

6. Papageorgiou MS, Dadakas G, Donev K. Liposarcoma of the Spermatic Cord: a Case Report. Case Rep Med. 2011;2011:197584.

7. Doyle LA. Sarcoma Classification: an Update Based on the 2013 World Health Organization Classification of Tumors of Soft Tissue and Bone. Cancer. 2014;120(12):1763-1774.

8. Ahmet P, Yucel AF, Aydin I, Sahin DA, et al. Paratesticular Liposarcoma: a Radiologic Pathologic Correlation. J Clin Imaging Sci. 2011;1:57.

9. Khoubehi B, Mishra V, Ali M, et al. Adult Paratesticular Tumours. BJU Int. 2002;90(7):707-715.

10. Fubiao L, Tian R, Yin C, et al. Liposarcoma of the Spermatic Cord Mimicking a Left Inguinal Hernia: a Case Report and Literature Review. World J Surg Oncol. 2013;11:18.

11. Khandekar MJ, Raut CP, Hornick JL, et al. Paratesticular Liposarcoma: Unusual Patterns of Recurrence and Importance of Margins. Ann Surg Oncol. 2013;20(7):2148-2155.

12. Filip V, Zejnilović N, Ivović J. Liposarcoma of the Paratesticular Tissue and Spermatic Cord: a Case Report. Vojnosanit Pregl. 2013;70(7):693696.

13. Mohammed R, Vinay HG, Ali M, et al. Bilateral Paratesticular Liposarcoma - a Rare Case Report. J Surg Tech Case Rep. 2014;6(1):1517.

14. Cerda T, Martin E, Truc G, et al. Safety and Efficacy of IntensityModulated Radiotherapy in the Management of Spermatic Cord Sarcoma. Cancer Radiother. 2017;21(1):16-20.

15. Kalaivani V, Hosamath V, Honnappa S, et al. Paratesticular LiposarcomaMasquerading as a Testicular Tumour. J Clin Diagn Res. 2014;8(2):165166.

16. Dayron R, Olumi AF. Management of Spermatic Cord Tumors: a Rare Urologic Malignancy. Ther Adv Urol. 2012;4(6):325-334. 